\title{
Multielectrode Evidence for Spreading Activity Across the Superior Colliculus Movement Map
}

\author{
NICHOLAS L. PORT, MARC A. SOMMER, AND ROBERT H. WURTZ \\ Laboratory of Sensorimotor Research, National Eye Institute, National Institutes of Health, Bethesda, Maryland 20982-4435
}

Received 27 December 1999; accepted in final form 11 April 2000

Port, Nicholas L., Marc A. Sommer, and Robert H. Wurtz. Multielectrode evidence for spreading activity across the superior colliculus movement map. $J$ Neurophysiol 84: 344-357, 2000. The monkey superior colliculus (SC) has maps for both visual input and movement output in the superficial and intermediate layers, respectively, and activity on these maps is generally related to visual stimuli only in one part of the visual field and/or to a restricted range of saccadic eye movements to those stimuli. For some neurons within these maps, however, activity has been reported to spread from the caudal SC to the rostral SC during the course of a saccade. This spread of activity was inferred from averages of recordings at different sites on the SC movement map during saccades of different amplitudes and even in different monkeys. In the present experiments, SC activity was recorded simultaneously in pairs of neurons to observe the spread of activity during individual saccades. Two electrodes were positioned along the rostral-caudal axis of the SC with one being more caudal than the other, and 60 neuron pairs whose movement fields were large enough to see a spread of activity were studied. During individual saccades, the relative time of discharge of the two neurons was compared using 1 ) the time difference between peak discharge of the two neurons, 2) the difference between the "median activation time" of the two neurons, and 3) the shift required to align the two discharge patterns using cross-correlation. All three analysis methods gave comparable results. Many pairs of neurons were activated in sequence during saccade generation, and the order of activation was most frequently caudal to rostral. Such a sequence of activation was not observed in every neuron pair, but over the sample of neuron pairs studied, the spread was statistically significant. When we compared the time of neuronal activity to the time of saccade onset, we found that the caudal neuronal activity was more likely to be before the saccade, whereas the rostral neuronal activity was more likely to be during the saccade. These results demonstrate that when individual pairs of neurons are examined during single saccades there is evidence of a caudal to rostral spread of activity within the monkey SC, and they confirm the previous inferences of a spread of activity drawn from observations on averaged neuronal activity during multiple saccades. The functional contribution of this spread of activity remains to be determined.

\section{IN T R O D U C T ION}

Maps of sensory and motor functions located on two-dimensional sheets of neurons are one of the fundamental organizational features of the brain. Within these sensory and motor maps, the activity is centered at one point; it is limited to a region that represents a restricted part of the sensory periphery

Address for reprint requests: N. L. Port, Laboratory of Sensorimotor Research, National Eye Institute, Bldg. 49, Rm. 2A50, Bethesda, MD 20982-4435 (E-mail: nlp@1sr.nei.nih.gov). or a restricted range of movements. A recent series of experiments on the cat and monkey superior colliculus (Munoz and Wurtz 1995b; Munoz et al. 1991a,b), however, suggests that such representations might not always be the case.

The superior colliculus (SC) has maps for visual input in the superficial layers and movement output in the intermediate layers. While the activity of most neurons on these maps is also essentially localized, with neurons in one part of the visual map being activated by a visual stimulus in one part of the visual field and neurons in the corresponding part of the movement map being activated before a saccadic eye movement to that stimulus, this is not the only type of activity observed. Munoz, Guitton, and Pellison (1991a,b) found that activity in the cat was not limited to one part of the SC map but instead moved across the SC from the caudal region (representing large saccades) to the rostral pole (representing small saccades) during saccade generation. The authors referred to this finding as a moving hill of activity. Munoz and Wurtz (1995b) found a more limited spread of activity in the monkey SC during saccade generation. They found that as a saccade progressed, activity that was prominent in the caudal SC began to extend more rostrally and began to do so before saccade initiation. Thus the dynamic spread of activity on the SC movement maps in both cat and monkey is strikingly different from the localized activity observed on the classically described maps of the $\mathrm{SC}$, as well as on maps in other regions of the primate brain.

The spread of activity in the monkey was limited to only one of several types of saccade-related neurons within the intermediate layers of the SC, namely, those referred to as having open-ended movement fields (Munoz and Wurtz 1995b). Neurons that have open-ended movement fields discharge before any saccade in a given direction that is larger than a given amplitude and usually are of the type referred to as buildup neurons (Munoz and Wurtz 1995a). Neurons with closed movement fields, on the other hand, increase their discharge rate only before saccades of a given optimal direction and amplitude and show no change in activity prior to saccades of substantially larger or smaller amplitudes and usually are of the type referred to as burst neurons (Munoz and Wurtz 1995a).

The key point in considering the spread of activity across the SC map during saccade generation is that for saccades with larger and larger amplitudes beyond the optimal for the particular neuron, the discharge occurs later and later during

\footnotetext{
The costs of publication of this article were defrayed in part by the payment of page charges. The article must therefore be hereby marked "advertisement" in accordance with 18 U.S.C. Section 1734 solely to indicate this fact.
} 
A

\section{Caudal Group}
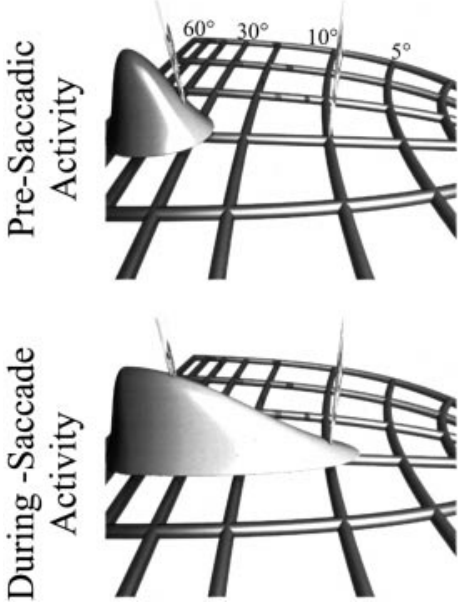

B In Between Group
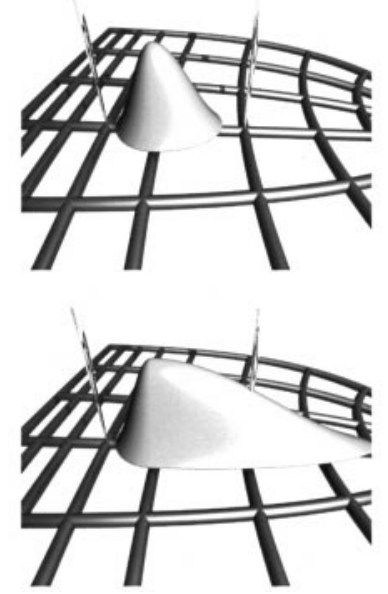

C

Rostral Group
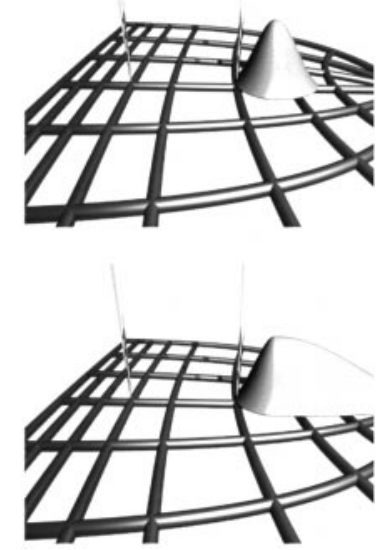

FIG. 1. Logic of determining whether there is a spread of activity across the superior colliculus (SC) using 2 electrodes at different positions on the SC map. The grid in each section is a 2-dimensional representation of the SC surface in polar coordinates with the rostral SC to the right and the caudal to the left. The mounds on the map represent the activity of a population of open-ended movement field neurons. Each row of the figure shows this activity before the saccade (top row) and during the saccade (bottom row). The 2 electrodes illustrated are at 10 and $30^{\circ}$ along the horizontal meridian. In the caudal group of saccades $(A)$, the hypothetical spread of activity is shown for a $50^{\circ}$ saccade, and because the activity begins at the caudal electrode, we would expect to see sequential activity on the 2 electrodes. In the in between group $(B)$, during a $20^{\circ}$ saccade, the activity would begin between the 2 electrodes, and the interpretation would be ambiguous. In the rostral group $(C)$, during a $7^{\circ}$ saccade, no evidence of spread would be expected. Our analysis concentrated on the case represented by the caudal group of saccades $(A)$ to examine whether there is evidence of sequential activity during the generation of a saccade and thus a spread of activity.

saccade generation. This increasingly later discharge of a given $\mathrm{SC}$ neuron as the saccade becomes larger formed the basis of the inference that there is a spread of activity across the SC map (Munoz and Wurtz 1995b), and observations supporting this inference were clearest for saccades of $20^{\circ}$ and larger. A subsequent study of this spread of activity concentrated on those saccades with amplitudes of $20^{\circ}$ or less (Anderson et al. 1998). Whereas a qualitative analysis of horizontal $20^{\circ}$ saccades resembled the results of Munoz and Wurtz (1995b), the quantitative analysis of $20^{\circ}$ horizontal saccades done by Anderson et al. (1998) showed no evidence of a significant spread and cast doubt on whether there is a spread of neural activity.

A major limitation of these studies in both the cat and the monkey is that the spread of activity is inferred from recordings of activity at different locations on the SC map during many saccades rather than from direct observation during single saccades. Furthermore, previous studies compared the neuronal activity across different saccades, on different days, and in different monkeys. In the present experiments we addressed these limitations by recording simultaneously from two neurons within the SC during individual saccades.

Figure 1 shows the logic of this experiment, and in particular the interaction expected between the location of the electrodes and the amplitude of the saccade. In Fig. 1A, the two microelectrodes are placed about 30 and $10^{\circ}$ along the horizontal meridian of the SC map, and the hypothetical spread of activity is shown for a $50^{\circ}$ saccade. The two panels are snapshots in time in which the mounds on the SC map represent the location of neuronal activity that is hypothesized to spread across the $\mathrm{SC}$ during the saccade. Because the activity begins on the SC map at (or more caudal to) the caudal electrode, we would expect to see sequential activity on the two electrodes. In contrast, if the activity is examined during a $20^{\circ}$ saccade (Fig. $1 B$ ), the sequence of activity expected is ambiguous, and if the activity is examined during a $7^{\circ}$ saccade (Fig. 1C), no evidence of spread would be expected. We therefore concentrated our analysis on the case represented in Fig. $1 A$ to examine whether there is evidence of sequential activity during the generation of a saccade and thus a spread of activity.

We found that many pairs of neurons were activated in sequence during saccade generation and that the order was most often caudal to rostral. Across the sample of neurons, the sequence of activation was caudal to rostral with the caudal neuronal activity tending to come before the saccade and the rostral neuronal activity tending to come during the saccade. These observations are consistent with a spread of activity across the SC but do not reveal what the function of such a spread might be.

A brief report has been published previously (Port et al. 1998).

\section{METHODS}

\section{Physiological procedures}

Two monkeys (Macaca mulatta) that weighed between 6 and $8 \mathrm{~kg}$ were prepared for chronic electrophysiological recording of single neurons and eye movements. Anesthesia was induced initially with intramuscular injections of ketamine HCL (10 mg/kg), diazepam (1 $\mathrm{mg} / \mathrm{kg})$, and glycopyrrolate $(0.01 \mathrm{mg} / \mathrm{kg})$. Monkeys were then intubated and maintained at a general anesthetic level with isofluorane. A subconjunctival eye coil was implanted (Judge et al. 1980) for recording eye movements. A plastic head holder for restraint and a chamber for microelectrode recording were secured to the top of the exposed skull with titanium screws and dental acrylic or antibiotic-embedded (cephazolin) bone cement (Palacos R). This hardware allowed subse- 
quent magnetic resonance images to be obtained. The trephine hole $(20 \mathrm{~mm})$ and overlying recording chamber were placed stereotaxically on the midline and angled $38^{\circ}$ back so that the electrode penetrations were directed caudorostral, toward the superior colliculus. At the end of surgery and one day postsurgery the animals were given flunixin meglumine $(2.0 \mathrm{mg} / \mathrm{kg})$ or buprenorphine $(0.005 \mathrm{mg} / \mathrm{kg})$ for analgesia and ketoprofen $(2 \mathrm{mg} / \mathrm{kg})$ for inflammation. An antibiotic, cephazolin $(30 \mathrm{mg} / \mathrm{kg})$, was given one day before surgery and every day for 14 days after surgery. Monkeys recovered for at least 1 wk before behavioral and physiological recording commenced. All protocols were approved by the Institute Animal Care and Use Committee and complied with Public Health Service policy on the humane care and use of laboratory animals.

Eye movements were recorded using the magnetic search coil technique (Fuchs and Robinson 1966). Single neurons were recorded with tungsten microelectrodes (Frederick Haer) with impedances between 0.7 and $1.5 \mathrm{M} \Omega$ measured at $1 \mathrm{kHz}$. Electrodes were aimed toward the superior colliculus through stainless steel guide tubes held in place by a plastic grid that was secured to the recording chamber (Crist et al. 1988). Two or three electrodes were used in each recording session, and each was attached to an individual drive that was all mounted to a metal ring atop the implanted recording cylinder.

\section{Behavioral procedures}

All behavioral paradigms were presented, and all data were collected and stored on a 486 personal computer running a QNX-based real time system that controlled the monkey's behavior and stored the experimental data (REX) (Hays et al. 1982). Action potential waveforms from individual neurons on each of the electrodes were amplified and identified with a dual-window discriminator using a 16channel integrated, computer-controlled DSP system (Plexon) that returned a pulse for each identified action potential to the REX system. The Plexon system also allowed us to record from multiple neurons from the same electrode. The time of occurrence of each action potential was stored with $1-\mathrm{ms}$ resolution. Horizontal and vertical eye positions were digitized at $1 \mathrm{kHz}$.

For the duration of the recording sessions (3-6 h), the monkeys were seated in a primate chair with the head restrained. The monkeys had an unobstructed view of $70 \times 70^{\circ}\left( \pm 35^{\circ}\right.$ from center in any direction) of a tangent screen positioned $57 \mathrm{~cm}$ in front of them. Each behavioral trial lasted $\sim 2-3 \mathrm{~s}$ and was preceded by an initial 2- to 3 -s period in which the screen was diffusely illuminated $\left(1.0 \mathrm{~cd} / \mathrm{m}^{2}\right)$ and the monkey was not required to fixate. At the start of each behavioral trial, the background light was extinguished and the task was performed in total darkness except for the presence of the back-projected red target spots produced by lasers. The light/dark cycle prevented the monkey from becoming dark adapted. The position of the projected laser beam was controlled by an X-Y mirror galvanometer system (General Scanning, M3 and CSX660). The first target spot, referred to as the fixation point, came on $250 \mathrm{~ms}$ after the monkey was in the dark. The monkey had to look at the fixation point and maintain fixation for 1.0-1.5 s before making a saccade to a second target. The task was a visually guided saccade task so that the target appeared in the peripheral visual field at the same time that the fixation point was turned off

To use the linear range of the eye coil system for larger saccades, the location of the fixation point on each trial was shifted so that the saccade was made across the central region of the field. For $40^{\circ}$ horizontal saccades, for example, the fixation point was moved left $20^{\circ}$ from center so that the monkey made a $40^{\circ}$ saccade across the center of the screen to the target positioned $20^{\circ}$ to the right. This shift of fixation point position was done for all saccadic amplitudes of $10^{\circ}$ or larger.

During the period of fixation before the saccade, the monkey was required to maintain eye position within a computer-controlled window that was \pm 1 to $\pm 5^{\circ}$ in both the horizontal and vertical positions.
For the saccade targets, a small window $\left( \pm 1^{\circ}\right)$ was used for small distances from the center of the screen; larger windows were used for larger amplitude targets. If the monitored eye left this window, the trial was aborted and the monkey received no reward. The monkey was allowed up to $500 \mathrm{~ms}$ to initiate the saccade after the target had been illuminated, and an additional $500 \mathrm{~ms}$ to enter the computercontrolled window around the target. If both of these conditions were not met, the trial was aborted and no reward was delivered.

A monkey typically performed between 1,500 and 3,000 trials in the experimental period as it worked to satiation for water rewards. It was then returned to its home cage. Records were kept of the weight and health status of the monkeys, and supplemental fruit and water were provided as needed. The monkeys were under the care of the institute veterinarian

\section{Neuron selection and analysis}

In each experimental session, two or three independently controlled electrodes were advanced into separate guide tubes directed at previously identified regions along a rostral-caudal line in the SC. Using the visually guided saccade task described above, the two or three electrodes were advanced one at a time until saccade-related neurons were isolated on each. We then cycled around the electrodes attempting to identify neurons that met two criteria: the location of the movement field was close to the horizontal meridian of the SC and movement fields were large (see RESULTS). If neurons with both these characteristics were not found on a given electrode, the electrode was either removed or repositioned and advanced again. Not until at least two electrodes were recording well-isolated neurons with the appropriate characteristics was the experiment begun. It was not unusual to spend at least three-quarters of the time and rewards during a session on the positioning of the electrodes, and thus we never successfully obtained appropriate neurons on all three electrodes.

To examine a spread of activity, it was important to have the pair of electrodes arranged along a single iso-directional line from the rostral pole along the collicular map. The rostral electrode was aimed at between 5 and $20^{\circ}$ along the horizontal meridian, and the caudal electrode was aimed at between 20 and $40^{\circ}$ along the same meridian. Such an alignment of both electrodes was difficult to achieve using the 1-mm grid system for the guide tubes (Crist et al. 1988). We therefore slightly bent the tungsten electrode shaft of both electrodes (roughly $1 \mathrm{~mm}$ curve for the last $10 \mathrm{~mm}$ of the electrode shaft). By rotating the direction of the bend before putting the electrode into the guide tube (using a similarly directed bend at the top of the electrode as a guide), it was possible to place both electrodes along the horizontal meridian. The direction of the curve was critical; on average each day at least one additional penetration was required in order for both electrodes to lie along the horizontal meridian.

Once the appropriate neurons were isolated that had their visual and movement fields along the horizontal meridian, their activity during a series of visually guided saccades was recorded. Saccades were made to eight target eccentricities $\left(3,5,10,20,30,40,50\right.$, and $60^{\circ}$ from the point of fixation) presented in a randomized block design. The eight target eccentricities were randomized within each block, and error trials were repeated in random order at the end of each block. Each block was then repeated usually 20 times for each pair of neurons recorded.

In off-line analysis, we determined whether the mean neuronal activity over the 20 trials showed a significant change before saccades to each of the eight targets. For neuronal activity to be considered significant for a given target, two criteria had to be met: 1) the peak-firing rate had to be greater than the mean plus 3 times the standard deviation of the background-firing rate in a 200 -ms period during fixation but before target onset, and 2) the peak firing rate for a given target had to be at least $20 \%$ of the peak firing rate of the best target for the given neuron. The second criterion was needed because a single action potential around the time of a saccade would meet the 
first criterion for those neurons that were inactive during fixation, but we did not believe such a small amount of activity should be considered saccade-related activity. The peak firing rates were derived from the average spike density function (see the next section) calculated for all of the saccades made to each target.

The size of the movement field for each neuron was taken as the difference between the amplitude of saccades to the smallest and the largest target eccentricity for which significant activity was found. Since the monkeys did not make accurate saccades to targets with large eccentricities, the mean saccadic amplitude for each target was used instead of the location of the target in estimating movement field size.

We searched for neurons that had open movement fields, those that had an increase of activity before a saccade of a given amplitude and continued to show increased activity before larger saccades. For example, such a neuron that had its maximal increase in activity before a $10^{\circ}$ saccade in a given direction should continue to increase its discharge rate before saccades of all larger amplitudes in that direction. This is in contrast to neurons with closed movement fields, which would discharge before the $10^{\circ}$ saccade but not before substantially larger saccades. Logically, one could only see a spread of activity in the neurons with such large movement fields because the spread means that a neuron at a given point on the SC map is active during saccades that are larger than the optimal for that point on the map. It is in these neurons that a spread of activity had previously been identified (Anderson et al. 1998; Munoz and Wurtz 1995a). It should be noted that we sampled movement fields only out to $60^{\circ}$, the maximum we could do with head-fixed monkeys. Thus it is possible that an open movement field would be closed if the monkey made gaze shifts $>60^{\circ}$. We did not study neurons that had a tonic discharge during fixation.

Because we were interested in the spread of activity within the SC, we converted these movement field sizes from visual field coordinates, which have a logarithmic relation of the visual and movement maps to eccentricity, into SC coordinates, which have a linear relation between distance on the SC and eccentricity (Robinson 1972). To do this we used the logarithmic transformation described by Ottes and colleagues (1986). This transformation allowed us to compare different movement fields centered at different eccentricities in the visual field on the linear SC map and thereby avoid the complexity of comparing different field sizes at different eccentricities on the logarithmic scale of the visual field.

The single spike activity on each trial was converted into a continuously varying spike density function (MacPherson and Aldridge 1979). Large values of the spike density function represent a greater probability of the occurrence of a spike, and the peak of the function represents the peak discharge of the cell. To generate the spike density function for each trial (Richmond et al. 1987), a Gaussian pulse of fixed width was substituted for each spike, and then all of the Gaussians were summed together to produce a continuous function in time. In this study the standard deviation of the Gaussian pulse $(\sigma)$ was 10 $\mathrm{ms}$. To maximize the advantage of recording from two neurons simultaneously, all of our analyses of activity of neuron pairs compared activity between the two neurons on a single trial.

All data were aligned to saccade onset that was defined as the point in time at which eye velocity reached a threshold $>30^{\circ} / \mathrm{s}$, assuming the eye also reached an acceleration $>8,000 \% \mathrm{~s}^{2}$. Single trials were omitted from any analysis if an error occurred in saccade detection due to small eye movements, eye coil noise, or other anomalies in the eye traces. Out of 13,044 trials, 174 were omitted from the analyses on this basis.

Standard statistical analyses (Snedecor and Cochran 1989) were used to analyze the data; the 0.01 probability level was taken as the level of statistical significance for rejecting the null hypothesis.
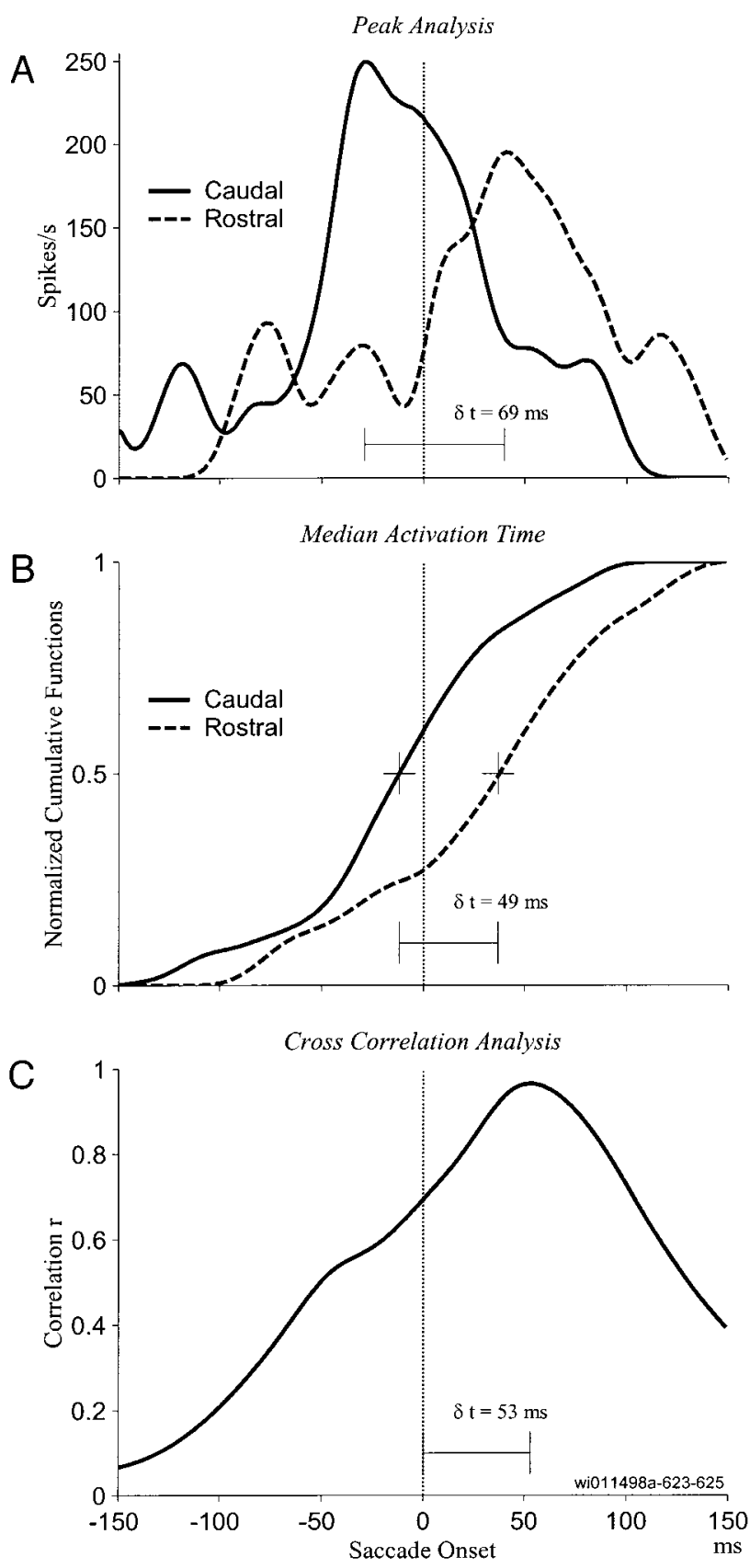

FIG. 2. Three methods of analysis for detecting spread of activity used in these experiments: peak analysis $(A)$, median activation time $(B)$, and crosscorrelation analysis $(C)$. See METHODS for description. The eye movement for this single trial was a $40^{\circ}$ horizontal saccade.

\section{Analysis for spread of neural activity}

We used several methods of analysis to examine the magnitude of the spread of activity, each of which had advantages and disadvantages, but when considered together, we thought gave a compelling answer. The three methods used were peak analysis, median activation time, and cross-correlation analysis, and Fig. 2 illustrates these methods applied to the recording of two neurons at two points on the SC on a single trial for a single saccade to a $40^{\circ}$ target. We examined whether there was a spread of activity in a \pm 150 -ms window centered on saccade onset, and thus our analyses were preformed on $300 \mathrm{~ms}$ of data. We chose $\pm 150 \mathrm{~ms}$ to avoid any contamination of neuronal activity by activity related to corrective saccades, particularly when 
the primary saccade was toward the distant targets. In addition, if a secondary saccade did occur within $100 \mathrm{~ms}$ after our \pm 150 -ms window, the examination window was made smaller so that there was at least $100 \mathrm{~ms}$ between the end of the window and the correction saccade. For example, the examination window would be $\pm 130 \mathrm{~ms}$ if a secondary saccade occurred $230 \mathrm{~ms}$ after the primary saccade.

The first method was the peak analysis. In this analysis, which is similar to that performed by Munoz and Wurtz (1995b), the time of occurrence of the peak firing rate is found for each spike density function in a given trial. Figure $2 A$ shows the two single spike density functions recorded simultaneously. The solid line represents the caudal neuron, which is located at $38.3^{\circ}$ along the horizontal meridian of the superior colliculus in this example. The dashed line represents the rostral neuron, located at $9.7^{\circ}$ along the horizontal meridian of the superior colliculus. The caudal neuron peak occurs at $-29 \mathrm{~ms}$, and that of the rostral neuron at $+40 \mathrm{~ms}$. A negative value indicates that the peak occurred before saccade onset, whereas a positive value indicates that the peak occurred after saccade onset. To obtain a summary value for this trial, the difference is calculated between the time of occurrence of the two peaks: $+69 \mathrm{~ms}$ in this example. A positive difference between the two electrodes indicates a caudal to rostral spread of neural activity; a negative difference indicates a rostral to caudal spread of neural activity. The primary advantages of the peak analysis are that it allows direct comparison to a previous report (Munoz and Wurtz 1995b), is relatively simple, and is generally not affected by any increase in activity due to any early visual response of many of these saccade-related neurons. The primary disadvantage is the sensitivity of the analysis to noise that is exacerbated by the analyses based on single trials used in the present experiments. In addition, a peak in activity always can be found even in cases where no significant change in activity has taken place. We excluded target amplitudes if there was no significant activity on average (see above); nevertheless, individual trials occur in which a correct saccade is made and there is little or no saccade-related activity.

The second method was the median activation time analysis. This analysis locates the point in time at which half the spikes have occurred. First, a cumulative function of each spike density function is created (Fig. $2 B$ ), and the halfway point along the vertical axis of each cumulative function is then identified (Fig. $2 B$, plus signs). This is the point at which half of the neuronal activity has occurred within the time frame being analyzed $( \pm 150 \mathrm{~ms}$ centered on saccade onset; the amplitude of the 2 curves are normalized to one in Fig. $2 B$ for the illustration, but it is not necessary for the calculations). The center of activity for our example trial is $-12 \mathrm{~ms}$ for the caudal electrode and $+37 \mathrm{~ms}$ for the rostral electrode, and the difference is $+49 \mathrm{~ms}$ in this trial, which as in the peak analysis indicates a caudal to rostral spread. The primary advantage of this analysis is its robustness to noise even at the single trial level. In addition, in the case when there is no significant change in neuronal activity on a given trial, the center of activity falls at the middle of the range; this middle point is the time of saccade onset in our analysis, and the null hypothesis of no spread would be obtained. The primary disadvantage is that a strong early visual response of a neuron would move the halfway point earlier than would be the case if there were just a saccade-related response.

The third method was the cross-correlation analysis. In this analysis, a cross-correlation between the two spike density functions is performed, and Fig. $2 C$ shows the cross-correlation function for this single trial example. The correlation values between the two waveforms for all possible shifts of the waveforms in time are first calculated, and the peak of the resulting curve then indicates the time shift that produced the best correlation. In this example the best shift was $+53 \mathrm{~ms}$, which again indicates a caudal to rostral spread. The primary advantage of this analysis is that it also takes into account the whole waveforms. The primary disadvantages are that a cross-correlation can be derived from nearly flat, noisy neural activity, which as indicated above can occur occasionally even for saccades to targets that on average yield significant saccade-related activity, and the occurrence of a large visual response in one of the neurons can produce a shift different from that in the absence of a visual response.

\section{RE S U L T S}

\section{Neuron sample}

We isolated and maintained 109 pairs of neurons for the duration of the experiment. For 60 of these pairs, both neurons had large movement fields in which a spread of activity could be studied, and subsequent analysis was performed on this set of neurons.

The neuron pairs were distributed across much of the SC map. The scatter plot in Fig. 3A, lower left, shows the location of the rostral and caudal neuron for each pair of neurons with the location for the caudal neuron of each pair on the abscissa and the rostral one on the ordinate. The scale is in millimeters, from rostral $(0 \mathrm{~mm}$ on the scale $)$ to caudal $(-4.5 \mathrm{~mm})$ locations of the SC. We converted to this distance scale on the SC to convert the recording locations from the logarithmic mapping of a neuron's strongest activity in degrees of visual angle (polar coordinates) to the linear millimeter scale of the SC map. For ease of reference, the SC map in the top right of Fig. $3 A$ shows both polar coordinate degrees of rotation and eccentricity as well as the collicular distances in millimeters. The histograms along the axes in Fig. $3 A$ show the frequency of neurons found at each SC location and indicate that over the sample of neuron pairs there was a substantial range of rostral to caudal locations represented. The median position of the rostral electrode was $-1.41 \mathrm{~mm}$, and the median for the caudal electrode was -2.87 $\mathrm{mm}$.

There is one region of the SC map that is underrepresented in the sample: the area within approximately $0.5 \mathrm{~mm}$ of the rostral pole, which represents saccades of less than about $2^{\circ}$ in amplitude. In this region we were unable to find neurons that had open-ended movement fields, that is, neurons that discharged with saccades of $<2^{\circ}$ amplitude and continued to do so for saccades that were substantially larger. This was not because we did not attempt to isolate open-ended movement field neurons in this region but rather because we failed to find them. It is important to note that this is a qualitative finding since during the experiment we determined whether the movement field was open or closed using the on-line raster display, not the statistical test of significance used in the off-line analysis.

Figure $3 B$ shows the range of saccades for which each neuron in the sample had a significant increase in activity, that is, the extent of the movement field of the neuron along the horizontal meridian of the visual field. The abscissa shows the estimated location of the cell on the SC map. The ordinate shows the rostral and caudal extent of the movement field along the horizontal meridian (top and bottom of each line, respectively); the center of all movement fields were within $5^{\circ}$ of the horizontal meridian. Because we searched for neurons in which a spread of activity had previously been identified, namely those with open-ended movement fields, the line for each neuron in Fig. $3 B$ should run from some point on the SC map representing the rostral end of the field (top of each line) to the caudal most $\mathrm{SC}$ region (bottom of each line beyond the $-4 \mathrm{~mm}$ point). For example, the neuron represented at the far left of Fig. $3 B$ has a movement field that starts at about 0.75 
A

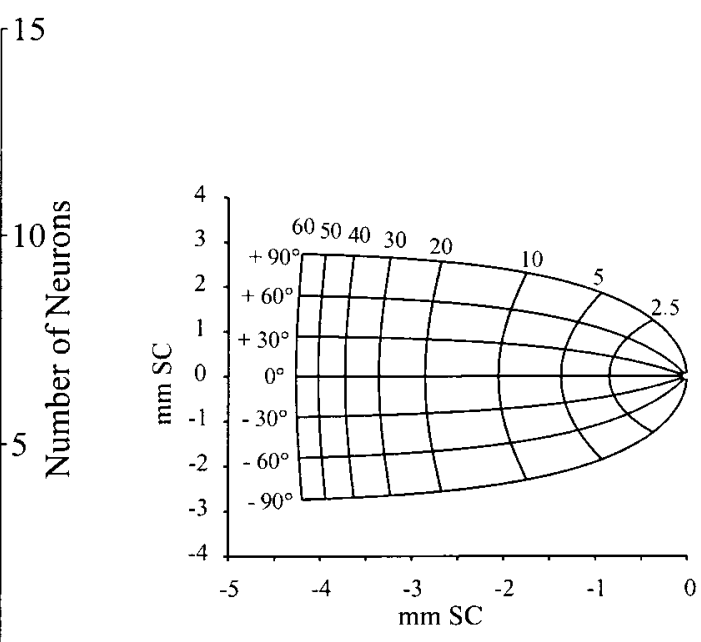

Caudal Electrode Location (mm)

$\begin{array}{llllllllll}-4.5 & -4 & -3.5 & -3 & -2.5 & -2 & -1.5 & -1 & -0.5 & 0\end{array}$

0

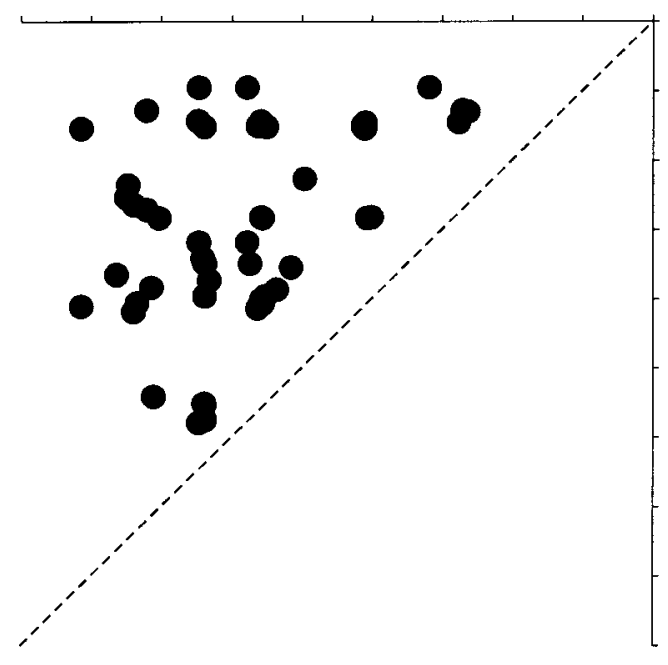

0

0

Number of Neurons

B

Electrode Location ( $\mathrm{mm}$ )

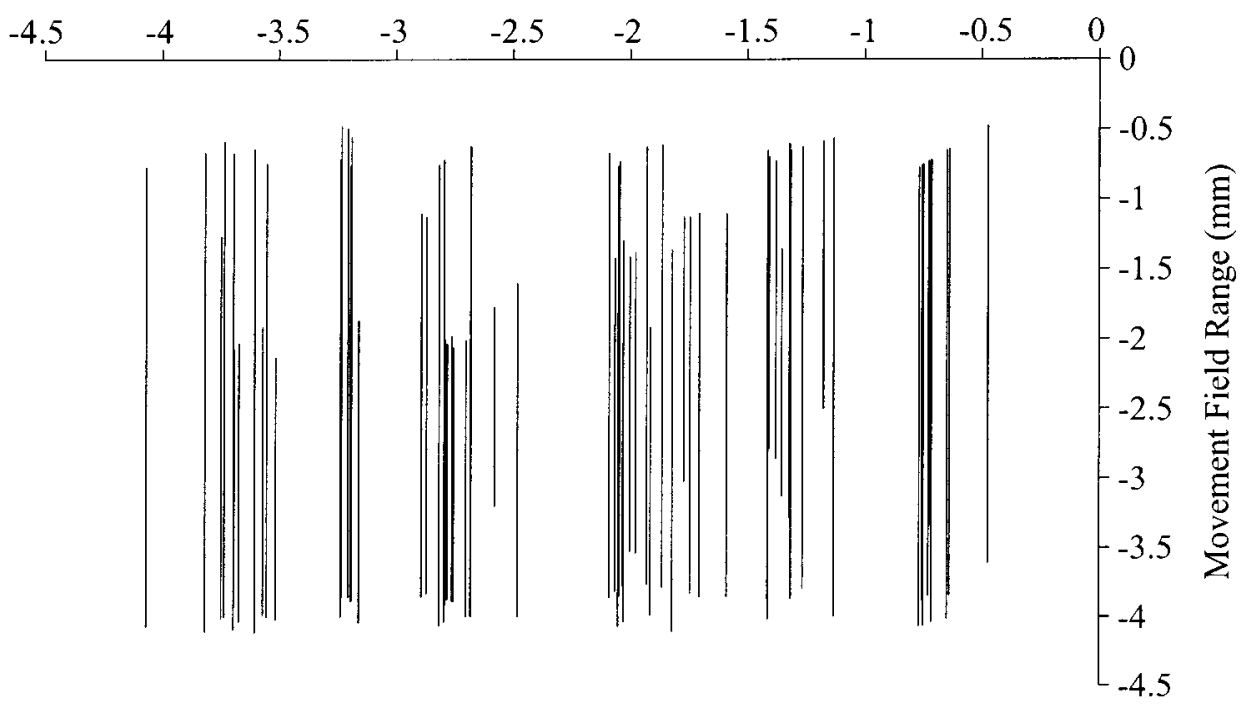

FIG. 3. Distribution of neuron pairs across the SC map. A: a scatter plot for each of the 60 neuron pairs with the location of the caudal neuron on the abscissa and the rostral one on the ordinate. The histograms show the frequency of neurons at different SC locations sampled at each $0.25 \mathrm{~mm}$ of SC rostral-caudal length. Inset, top right: SC map with both degrees of the visual field in polar coordinates and $\mathrm{mm}$ on the SC map indicated for comparison. B: the range of saccades before which each neuron in the sample showed activity significantly different from the background activity. Each neuron is plotted according to its location on the SC map. 

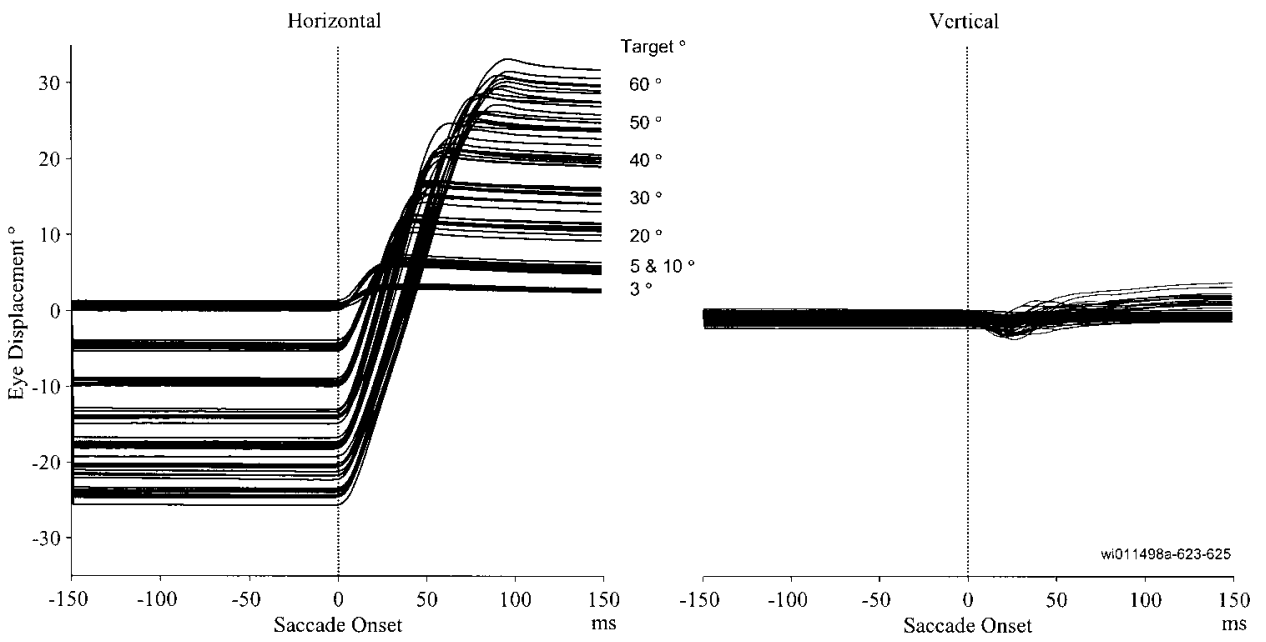

FIG. 4. Saccadic eye movements to a series of 8 targets from 3 to $60^{\circ}$ on the horizontal meridian that were made while the sample pair of neurons shown in Fig. 5 were recorded. The vertical dashed line indicates saccade onset. Note the negative horizontal starting position of the eye, which indicates the location of the fixation point, as well as the negligible amount of vertical displacement of the eye. $\mathrm{mm}$ on the SC map and extends beyond $-4 \mathrm{~mm}$ on the map. We encountered other neurons, however, that had large movement fields but did not extend all the way to the largest saccade represented on the SC map $(-4 \mathrm{~mm})$. We included these larger field neurons in our sample even though they did not meet the strict criteria for an open-ended movement field of responding to all larger saccades because 1 ) their movement fields were substantially larger than typical burst neurons with closedended movement fields recorded at the same region of the SC, and 2) the movement fields of both neurons of a pair were always large enough to allow overlap of the fields of the caudal and rostral neurons and thus allow identification of any sequential activity between them. We accepted a movement field as being a large movement field if it was at least $1.33 \mathrm{~mm}$ in length along the rostral to caudal extent of the SC (for horizontal saccades). We chose $1.33 \mathrm{~mm}$ (33\% of the approximate $4 \mathrm{~mm}$ length of the SC) because that value makes these movement fields larger than previous estimates of closed movement fields. These previous estimates of closed movement field neurons were based on the observation that approximately $25-28 \%$ of the SC is active for a given saccade (Anderson et al. 1998; Munoz and Wurtz 1995b; Ottes et al. 1986). Although this size is arbitrary, using other numbers (e.g., 1.5 or $2 \mathrm{~mm}$ ) produced results over the sample that were nearly identical.

In the analysis that follows, we present the results in three steps. We will first provide an example of the analysis for the spread of activity using single pairs of neurons. This analysis is based on the difference in the time of activity of the caudal and rostral neurons, and this difference is derived from the comparison of the simultaneous activity at the two recording sites during each individual saccade. We will then show the extent of this spread across the entire sample of analyzed neuron pairs based on the mean time difference across all of the neuron pairs. Finally, we will examine the relation of the spread to saccade onset.

\section{Example of a simultaneously recorded neuron pair}

Figures 4-6 show the analysis of a pair of neurons that showed evidence of a spread of activity to illustrate both the nature of the observations on a single pair of neurons and the steps used in the analysis. After we isolated both neurons and

determined the centers of their movement fields, we recorded the activity of the neurons before saccades to a standard series of eight target eccentricities made along a line extending from the fixation point through the centers of the movement fields. Figure 4 shows the horizontal and vertical eye movement traces as a function of time for all eight targets $(3,5,10,20,30$,

Caudal Neuron
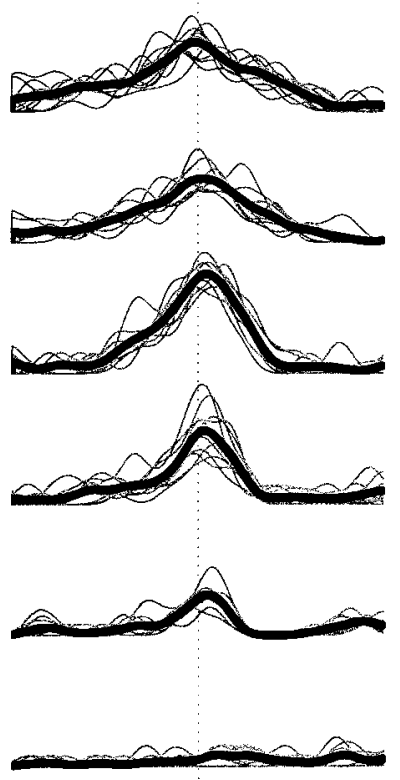
$10^{\circ} \star$
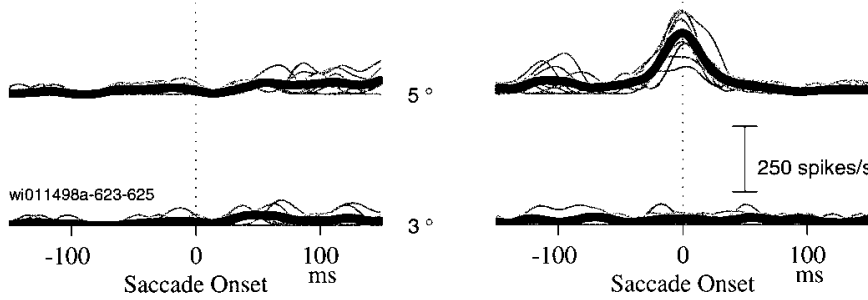

FIG. 5. Example of the activity of 2 neurons recorded at 2 points on the SC during the series of saccades shown in Fig. 4. The thin spike density functions are for individual trials $(\sigma=10 \mathrm{~ms})$, and the thick black line is the mean. Left: the caudal neuron. Right: the rostral neuron. The vertical dashed line indicates saccade onset, and the asterisks indicate those targets for which there was significant saccade-related activity for both neurons (see METHODS). 

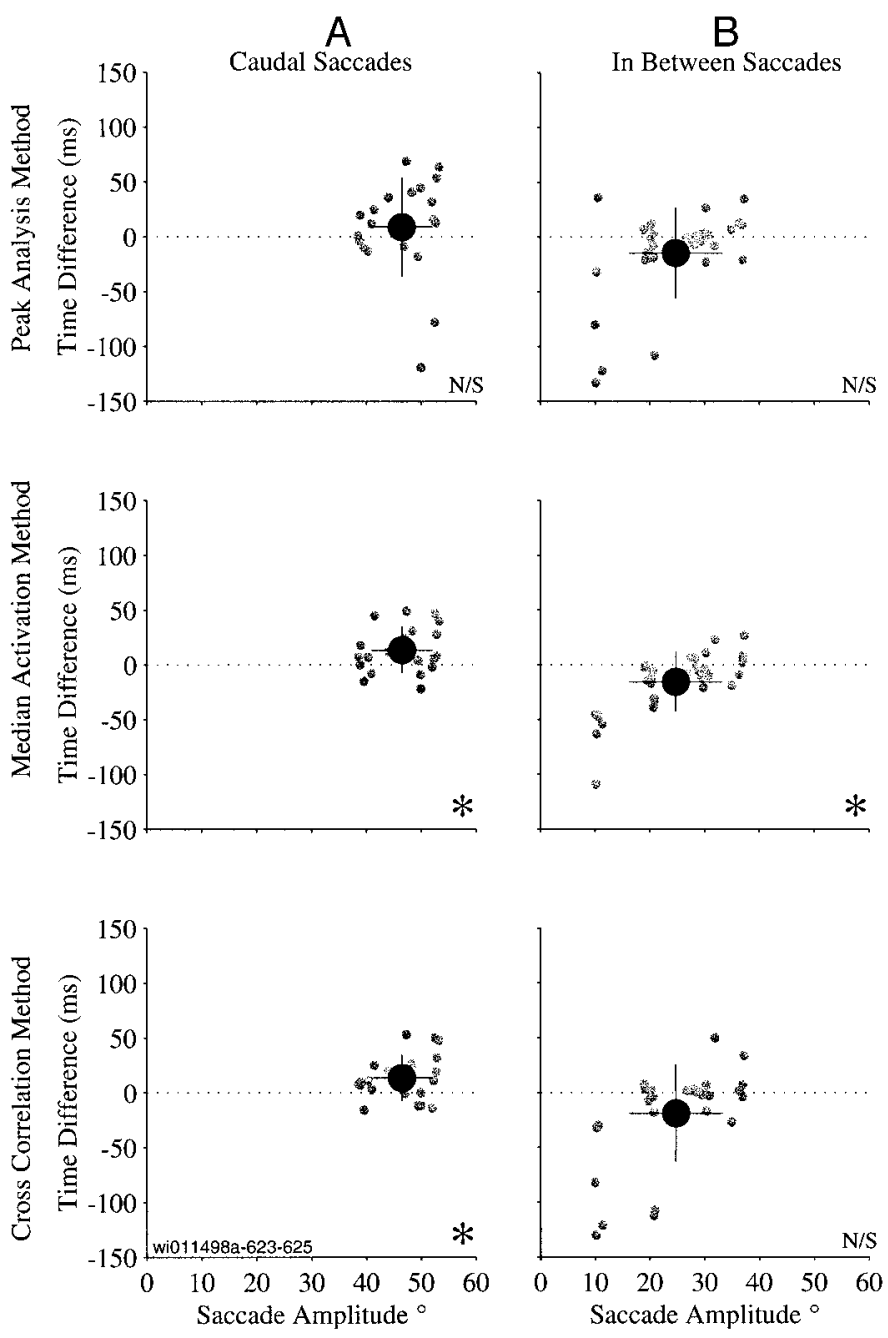

40,50 , and $\left.60^{\circ}\right)$. Note the negative horizontal starting position reflecting the location of the fixation point, and the limited vertical eye displacement indicating nearly horizontal saccades. The set of saccades also shows the variation in amplitude, particularly with larger saccades, that led us to plot neuronal activity with respect to saccade amplitude and not to target location.

Figure 5 shows the activity associated with these different amplitude saccades for the $150 \mathrm{~ms}$ before and after saccade onset (time 0 ) for the caudal and rostral neuron (left column and right column, respectively). The asterisks indicate the groups of saccades to a target in which the average activity on both the caudal and rostral electrodes showed a significant change in activity, and it was for these groups that comparisons of the timing of activity between neuron pairs were made. Note that the maximum activity of the caudal neuron occurs for saccades to the $40^{\circ}$ target, whereas the maximum activity for the rostral neuron occurs for those to the $10^{\circ}$ target.

As described in the introduction and Fig. 1, if there is a caudal to rostral spread of activity across the SC, then we would expect to see the clearest evidence of spread for saccades whose activity is associated with a site on the SC map at or more caudal to the caudal electrode of a pair; activity should then be seen spreading past the caudal and then the rostral electrode (Fig. 1A). Conversely, for those saccades associated
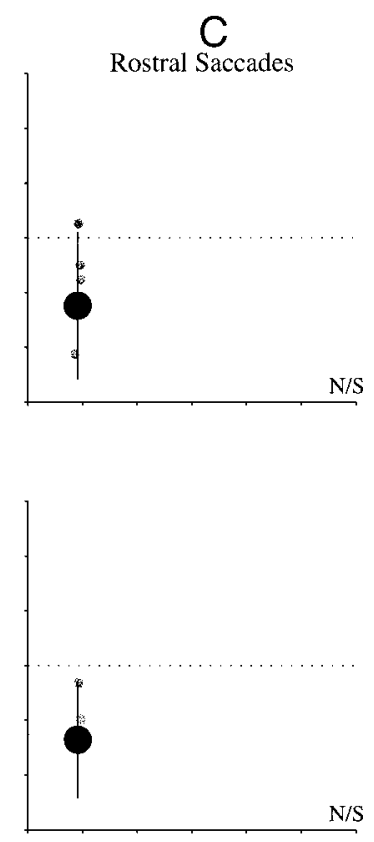

FIG. 6. Three measures of the spread of activity for the sample pair of neurons shown in Fig. 5. The measure of the spread (ordinate) was always in $\mathrm{ms}$, and this was determined using the peak analysis (top row), median activation time (middle row), and cross-correlation analysis (bottom row) as outlined in Fig. 2 and METHODS. The data for each analysis method were divided by saccade group into the caudal saccade group $(A)$, the in between saccade group $(B)$, and the rostral saccade group (C). The amplitude of the saccades is on the abscissa, and the time difference between the measure of caudal and rostral activity is on the ordinate. On all 3 plots, positive values indicate that the activity in the caudal neuron precedes that in the rostral neuron. Each small point is an individual trial; the large circle and bars are means \pm SD. In this sample pair the mean was significantly different from zero for the caudal saccade group in all 3 analysis methods $(t$-test, $P<0.01)$. The mean for the in between saccade groups was usually not significant and for the rostral group was never significant for any of the 3 analysis methods. The median activation time and cross-correlation analysis methods for the rostral saccade group were not significant $(P>0.01)$ even though the time differences were almost all negative. with SC sites rostral to both electrodes, the spread should be minimal or nonexistent (Fig. 1C); for saccades that originate in between the rostral and caudal electrodes the prediction is ambiguous (Fig. 1B). In light of these predictions, we divided our data set into three saccade groups; those whose associated neuronal activity was at or caudal to the caudal electrode (the "caudal group"), those with activity originating in between the two electrodes (the "in between group"), and those with activity rostral to the rostral electrode (the "rostral group").

Figure 6 shows the results for one neuron pair (same pair as in Figs. 4 and 5). The columns show the results for each of these three saccade groups, and the rows show the results using the three methods of analysis: peak activity, median activation time, and cross-correlation (as illustrated in Fig. 2 for the same neuron). The ordinate for each graph represents the time difference in milliseconds between caudal and rostral neurons. Since in all three methods the caudal value was subtracted from the rostral, a spread of activity from caudal to rostral is indicated by a positive value. The abscissa of each graph gives the amplitude of the saccade on each trial that yielded the time difference between the two neurons on the ordinate. Each small point indicates the time difference between the pair of neurons for a single saccade; the large black circle and bars indicate the mean and standard deviation of the points for that saccade group. This is the same neuron pair as in Fig. 4, so the caudal 

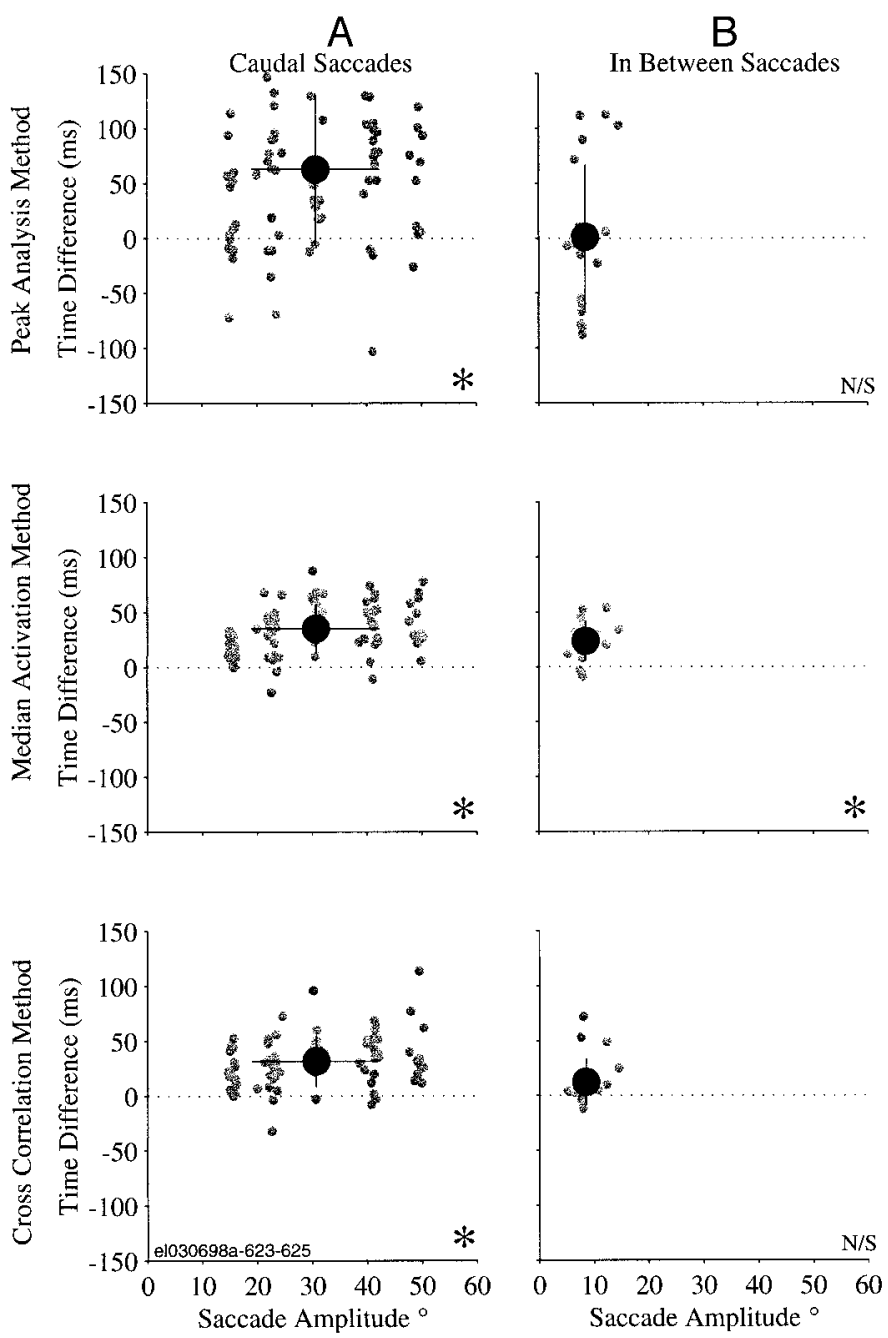

neuron had maximal activity with saccades to the $40^{\circ}$ target, although the mean amplitude of the saccades was $38.3^{\circ}$, and the rostral neuron had maximal activity before saccades to the $10^{\circ}$ target, and the mean amplitude of the saccades was $9.7^{\circ}$. The saccades to all of the eight targets that were placed in the caudal group had amplitudes of $38.3-53.3^{\circ}$, those in the in between group were $9.7-38.3^{\circ}$, and those in the rostral group were $<9.7^{\circ}$. Note that saccades to a given target can be split into two saccade groups depending on the amplitude of the saccade actually made.

The caudal group of saccades are of greatest interest (Fig. $6 A$ ), and all three analysis methods for this neuron pair show two characteristics. First, for nearly every individual saccade as well as for the mean, there is a difference between the time of activity for caudal and rostral neuron that is positive, indicating that the activity occurred on the caudal before the rostral electrode. This is consistent with a spread of activity from caudal to rostral in the SC. The mean of the time difference for the peak analysis is $8.8 \mathrm{~ms}$, for the median activation time analysis it is $13.5 \mathrm{~ms}$, and for the cross-correlation method it is $13.4 \mathrm{~ms}$. These means are significantly different from zero ( $t$-test, $P<0.01$ ) except for the peak analysis. The two methods that relied on the shift of the entire curve gave similar and statistically significant results. Second, there is substantial scatter in the time differences for individual trials, including some
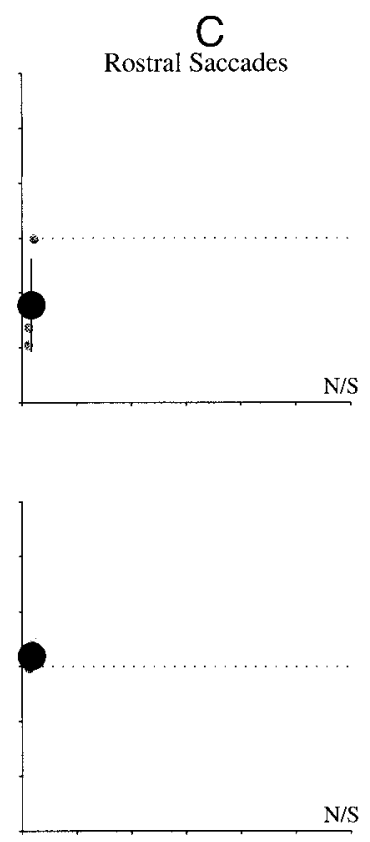

FIG. 7. Three measures of the spread of activity in a 2nd example pair of neurons. Same conventions as in Fig. 6.

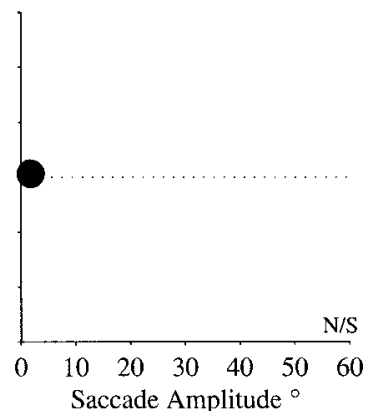

points that are clearly negative. Overall, this scatter is less using the median activation time $(\mathrm{SD}=21.1 \mathrm{~ms})$ and the cross-correlation methods $(\mathrm{SD}=20.9 \mathrm{~ms})$ than the peak analysis ( $\mathrm{SD}=45.2 \mathrm{~ms}$ ) as indicated by several disparate points consistent with its greater susceptibility to noise.

For the rostral saccade group (Fig. 6C), where we would expect minimal evidence for a spread of activity, the means are not significantly different from zero. As indicated in the INTRODUCTION, the interpretation of the in between saccade group is ambiguous, and as shown in Fig. $6 B$, the mean is not significantly different from zero ( $t$-test, $P>0.01$ ) except for the median activation method.

Figure 7 shows a second example of the activity of a pair of neurons for which there are more saccades in the caudal group; that is, the caudal electrode is more rostral in the SC with maximal activity at $14.6^{\circ}$ (at $3.7^{\circ}$ for the rostral neuron). Again there is clear evidence for a spread of activity in the caudal group (Fig. 7A) since the difference in timing between the caudal and rostral neuron is again positive: $62.9 \pm 69.0$ (SD) $\mathrm{ms}$ for the peak method; $35.2 \pm 21.9 \mathrm{~ms}$ for the median activation time method; $31.5 \pm 23.3$ for the cross-correlation method. Again the in between and rostral groups (Fig. 7, $B$ and $C$ ) generally did not have mean differences that were significantly different from zero.

In summary, for these sample pairs of neurons, there is clear 

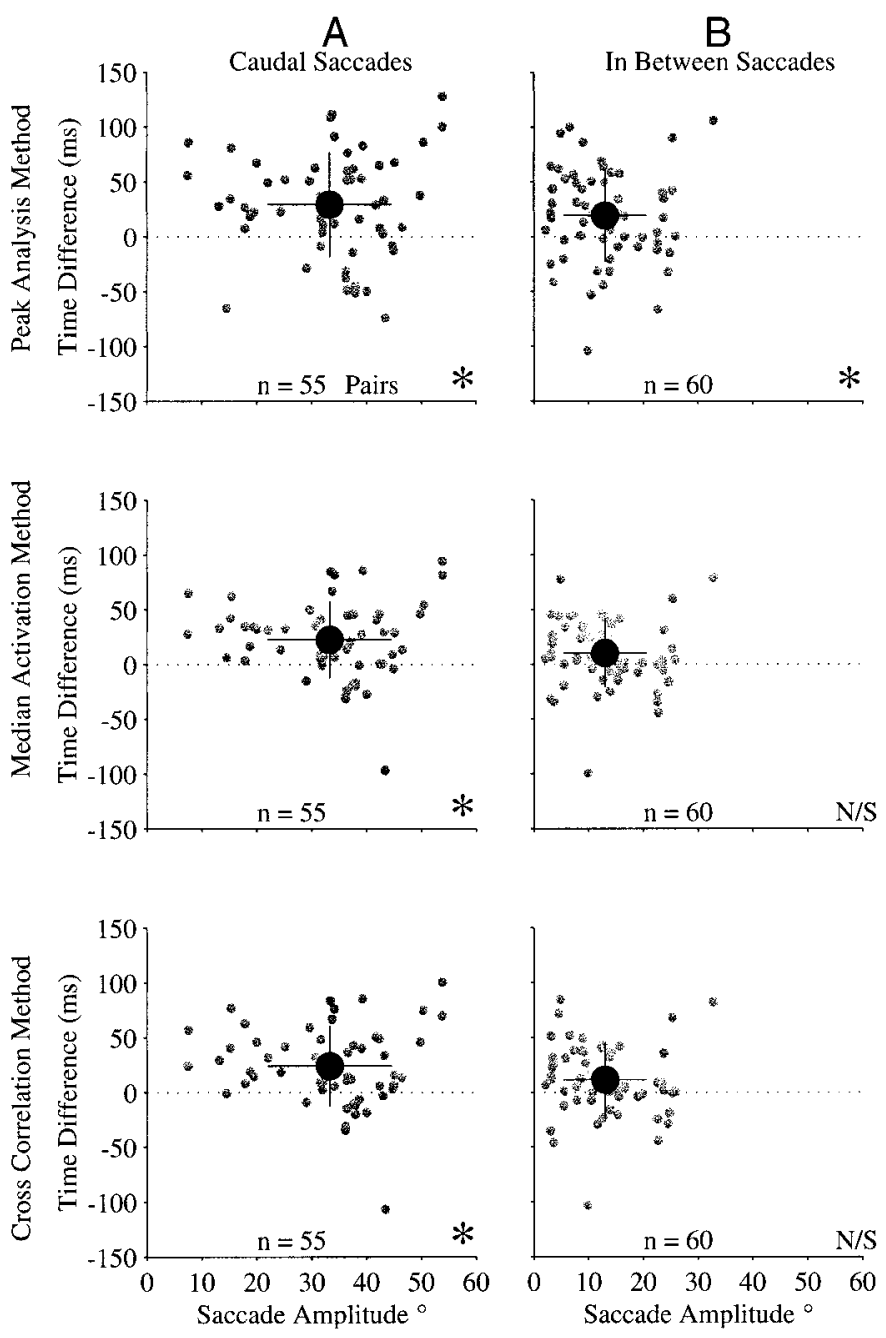

evidence of a difference in timing between the activity of the caudal and rostral SC neurons that is consistent with a spread of activity from caudal to rostral within the SC. The time difference in both cases was substantial, with best estimates ranging from 10 to $30 \mathrm{~ms}$.

\section{Spread in the sample of neurons}

We next examined the evidence for a spread of activity across the sample of the 60 neuron pairs. To do this we used the mean time differences for each neuron determined by each of the three methods. Figure 8 shows these mean time differences plotted for the three saccade groups and for the three analysis methods used in Figs. 6 and 7, but now each point represents the result for one neuron pair rather than one trial. The overall mean of these individual neuronal pairs means is again indicated by the large circle.

For the caudal saccade group in Fig. 8A, 55 of the 60 neuron pairs are included; those five that are not included did not have movement fields of the rostral and caudal neurons that overlapped. The most caudal location of the caudal electrode was at $-4.07 \mathrm{~mm}$, and the most rostral location of the caudal electrode was $-1.32 \mathrm{~mm}$ with a mean location of $-2.97 \mathrm{~mm}$. The first result shown in Fig. 8 is that, for the entire sample of neurons tested in the caudal saccade group, the mean of the
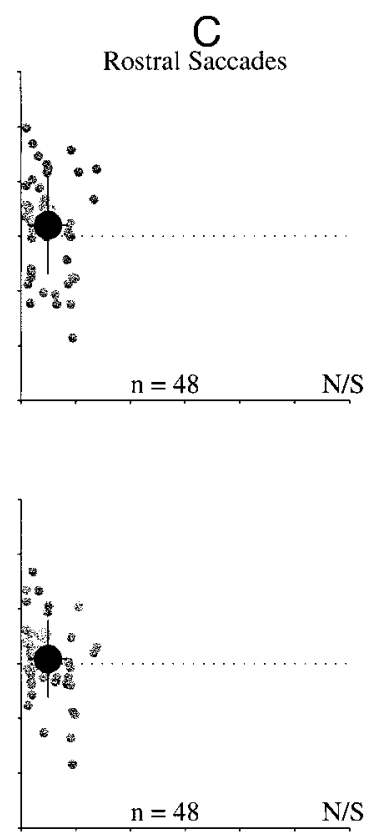

FIG. 8. The amount of spread for all 60 neuron pairs studied. Conventions similar to those in Fig. 6. The small circles are the mean time difference and saccade amplitude for each analysis method for each pair studied. The large circles and bars are the means \pm SD for the sample of neurons. For the caudal saccade group, the mean was significantly different from zero for all 3 analysis methods (*). For the rostral and in between saccade group, the mean was not significantly different from zero except for the peak analysis method with the in between saccade group. The $n$ is the number of neuron pairs in each saccade group.

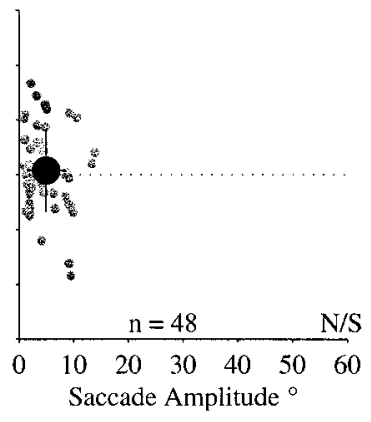

time difference between the rostral and caudal neuron in the sample was positive and significantly different from zero $(t$ test, $P<0.01)$. This indicates that in the pooled sample of 55 neuron pairs, there was a caudal to rostral spread of activity. The means were 29.3, 22.6, and $24.1 \mathrm{~ms}$ for the peak, median activation time, and cross-correlation method, respectively. Again, the mean values for the median activation and crosscorrelation methods were closer together than they were to the peak value, and we take the best estimate of the mean difference to be about $23 \mathrm{~ms}$.

There is a possible explanation for the increased activity on the rostral neuron that should be addressed. In this explanation, the activity on the rostral neuron results not from a spread of activity related to the saccade but instead from the visual input to the rostral neurons generated as the saccade sweeps the image of the target across the receptive field. We believe that this is unlikely because any such visual response should come substantially later than the spread of activity we have measured. In our laboratory the latency of the visual response to the relatively dim red laser spot stimuli is approximately $80 \mathrm{~ms}$. For the large saccades in our experiment, the target stimulus does not pass over the receptive field of the rostral neuron (which is close to the fovea) until near the end of the saccade. This would add another 10-30 ms to the latency of any visual response on the rostral neuron in relation to saccade onset, 


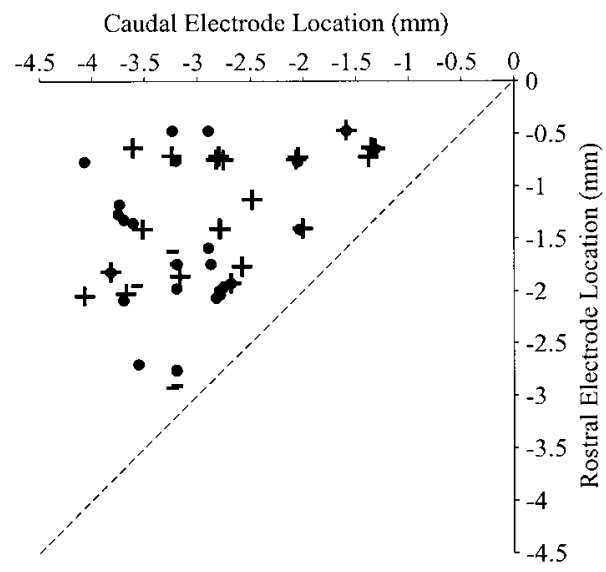

FIG. 9. Distribution of the sample of neuron pairs indicating which had values significantly different from zero signifying caudal to rostral spread $(+)$ or rostral to caudal spread (-). Other points represent pairs whose values were not significantly different from zero. All points are from the caudal saccade group shown in Fig. 8A using the cross-correlation analysis method and represent 55 neuron pairs. Plot organized as in Fig. $3 A$.

depending on saccade amplitude. Thus a visual response on the rostral neuron should be approximately $100 \mathrm{~ms}$, a value much longer than the mean time of the spread, $23 \mathrm{~ms}$, we report.

Since each point in Fig. $8 A$ represents the mean for a neuron pair, the plot also shows a second finding as well: many neuron pairs had little or no difference in the timing of the activity at the two neurons (those near the zero line), and some neuron pairs also had a negative value for this difference indicating that there was a rostral to caudal spread for these neuron pairs. The caudal to rostral shift represents a mean change in activity that does not occur in all neuron pairs.

Figure 9 shows where on the SC map the pairs of neurons were that had either a significant positive value indicating a caudal to rostral sequence of activity $(+)$ or a significant negative value indicating a rostral to caudal sequence $(-)$ ( $t$-test, as in Figs. 6 and 7, using the cross-correlation analysis method). Clearly there are many more positive $(n=24)$ than negative values $(n=5)$, and these positive values are spread out across the caudal to rostral extent of the SC map. The circles represent pairs in which the spread was not significantly different from zero.

For the in between saccade group (Fig. 8B, 60 neuron pairs), the mean was not significantly different from zero for the median activation and cross-correlation methods ( $t$-test, $P>$ 0.01 ) but was significantly different from zero for the peak analysis method ( $t$-test, $P<0.01$ ). It is worth noting that the variance of the time differences for the peak analysis method $(\mathrm{SD}=42.2)$ was approximately $38 \%$ greater than the median activation and cross-correlation methods ( $\mathrm{SD}=31.1$ and 33.2). For the rostral saccade group (Fig. 8C, 48 neuron pairs), the means did not differ from zero.

In summary, when saccades were made with amplitudes as large or larger than amplitudes represented by both the caudal and the rostral neurons, we found a robust difference in timing across the sample of neuron pairs consistent with a caudal to rostral spread of activity across the SC map. No such spread was evident in many neurons, and some showed activity consistent with a rostral to caudal spread of activity, but the average spread was in the caudal to rostral direction with a time difference of about $23 \mathrm{~ms}$.

\section{Relationship of spread to saccade onset}

Our evidence so far indicates that on average the activity of more caudal neurons precedes that of more rostral neurons, and from this time difference we infer that there is a caudal to rostral spread of activity. We now need to determine how the time of activity of the pairs of neurons is related to the onset of the saccade to determine whether the spread of activity at different sites on the SC map leads or lags the saccade. This can be addressed by comparing the time of activity of the caudal and rostral neurons with the onset of the saccades on a trial-by-trial basis. Figure 10 shows the relation of the activity of the caudal (Fig. 10A) and rostral (Fig. 10B) neurons in relation to saccadic onset for the caudal saccade group since it was that group that showed the clearest spread of activity. We used both the peak method $(-)$ and the median activation method $(\cdots)$; the cross-correlation method did not yield separate activity values for each neuron. The means of the two distributions for the peak analysis (Fig. 10, $A$ and $B$ ) were -20.8 and $8.2 \mathrm{~ms}$ for the caudal and rostral electrodes, respectively, and for the median activation analysis were -13.0 and $7.7 \mathrm{~ms}$, respectively. In both analyses the activity of the caudal neuron tends to be before saccade onset more frequently, and the activity of the rostral neuron tends to be during the saccade more frequently. This indicates that the spread of activity is largely due to the neuronal activity in the SC occurring later (i.e., during the saccade) in the rostral regions of the SC.

The time of the peak of the motor burst in the vast majority of our individual neurons occurred before the onset of the saccade made to the center of the movement field. In 22 of our

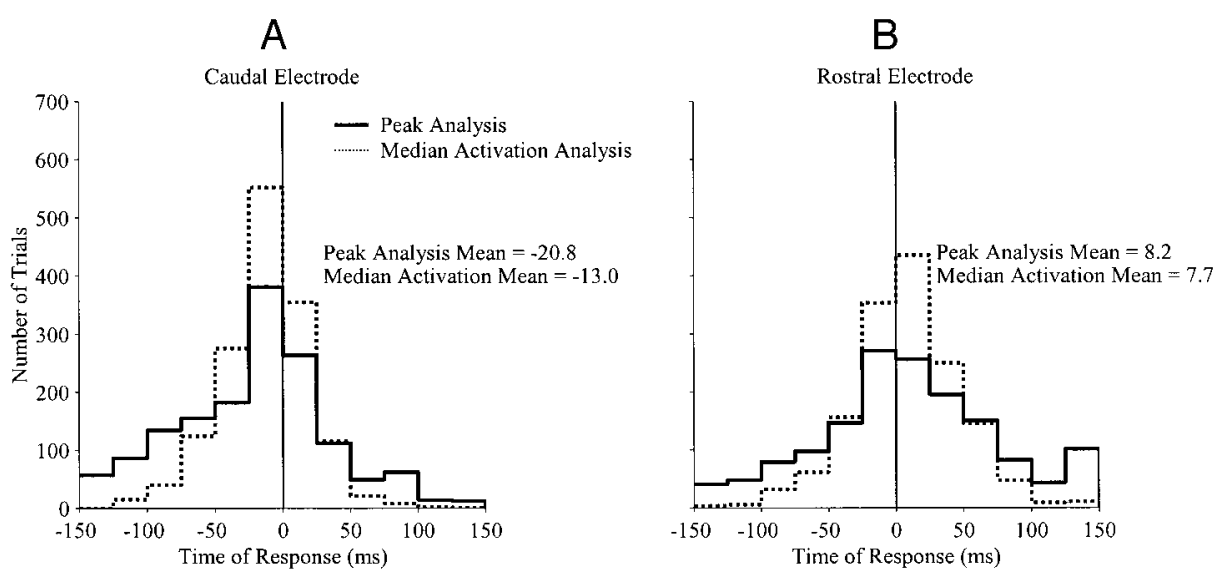

FIG. 10. Distributions of activity compared with the time of saccade onset for the caudal $(A)$ and rostral $(B)$ electrodes from the caudal saccade group in Fig. 8A. Data from every trial $(n=1,509)$ are shown. The peak discharge rate (-) and the distributions of the median activation times $(\cdots)$ show similar values. Note that the caudal values are more often before the saccade onset, whereas the rostral values are more likely during the saccade. 
120 individual neurons, however, the time of the peak of the motor burst was after the saccade onset. One possibility is that these few postsaccadic neurons contribute to the rostral to caudal spread in some neuron pairs shown in Fig. 8A. This could occur if we recorded from one of these postsaccadic neurons on the caudal electrode at the same time we recorded from the usual presaccadic neuron on the rostral electrode. This was not the case; such pre- and postsaccadic combinations were distributed across neuron pairs that showed caudal to rostral as well as those that showed rostral to caudal spread in the sample of neurons shown in Fig. 8A.

\section{I S C U S S I O N}

By recording simultaneously between pairs of SC neurons, we have been able to determine whether there is any sequential activation of two neurons located at different caudal-rostral sites during the generation of a saccade. We found that many pairs of neurons were activated in sequence during saccade generation and that the order was most often caudal to rostral. In the pooled sample of neurons, there was also clear evidence for a sequential activation of neurons from caudal to rostral. When we compared the time of neuronal activity to the time of saccade onset, we found that the caudal neuronal activity was more likely to occur just before the saccade, whereas the rostral neuronal activity was more likely to occur during the saccade. These observations are consistent with a spread of activity from caudal to rostral within the SC. In the sample of neuron pairs, however, there were some individual pairs that showed no such spread and a few that showed evidence of a rostral to caudal spread so that it is clear that the caudal to rostral spread is not seen between all neurons. There are a number of critical points in evaluating these observations that we will consider prior to discussing what the functional contribution of this spread of activity might be.

\section{Spread of activity and its limitations}

Our findings have both substantiated the original observation of a spread of activity in the monkey SC (Munoz and Wurtz 1995b) and revealed limitations of the phenomenon. First, the use of two electrodes allowed direct measurement of the sequence of activity of two individual neurons at two locations on the SC map during an individual saccade, whereas in previous experiments the successive activation of the rostral neuron after the caudal neuron was an inference from comparisons across the averaged activity of neurons at different locations on the SC map during saccades of the same average amplitude. The previous demonstration of the spread of activity in the experiments of Munoz and Wurtz (1995b) comes from recordings in four monkeys over thousands of different saccades over a period of several years, and a similar averaging in two monkeys is presented in Anderson et al. (1998). The present experiments based on the same two neurons in the same saccade converts the sequential activation from inference based on averages to observations on individual trials. Second, since we were recording from individual neurons during the same saccade, we could compare the time of activity of each to the saccade onset. Doing this showed that, for large saccades, more caudal neurons tend to be active prior to saccade onset and more rostral neurons during a saccade. This is also con- sistent with the inference that there is a caudal to rostral spread of activity. Finally, in the previous experiments of both Munoz and Wurtz (1995b) and Anderson et al. (1998), the placement of the recorded neuron on the SC map was critical because the placement determined the inferred sequence of activation; any errors in placement introduced errors in the apparent sequence of activation. The placement on the map, however, depends on the determination of the peak in a movement field (Munoz and Wurtz 1995b) or the visual receptive field (Anderson et al. 1998), neither of which necessarily has a sharp peak. In the present experiments, the neurons were also placed on the SC map using the movement field and so the same problem with localization exists in principle. But since the neurons were recorded at the same time during each saccade, it was possible to directly compare the activity of each neuron with each saccade. This direct comparison allowed determination of which neuron responded more vigorously to any given saccadic amplitude, and this in turn enabled us to place one neuron unequivocally either more rostral or more caudal than the other on the SC map. There could be no error in their relative caudal-rostral position on the SC map.

There are also several limitations to the current study. First, the spread of activity remains an inference because we were successful in recording just two neurons at a time. An irrefutable demonstration of spread would seem to require recording from a series of neurons in a caudal to rostral row, but we did not succeed in doing this largely because of the difficulty in isolating three neurons, all of which had large enough movement fields. On the other hand, the results do not lend support to the proposal of Anderson et al. (1998) that the sequential activation can be regarded as a combined fall of activity in the caudal colliculus and a rise at the rostral pole of the SC. For many neuron pairs in the present experiments, both the rostral and the caudal neurons were relatively remote from the rostral pole (see the example in Fig. 6 and the location of the pairs in Fig. 9), and yet there remained a sequence of caudal to rostral activation. Any rise and fall of activity at the rostral pole would be expected to affect both of these neurons similarly.

A second issue was that, while across the sample of neuron pairs there was a sequence of activation from caudal to rostral (Fig. 8), it was also clear that the scatter in the recorded points was substantial. This scatter included neuron pairs that showed little evidence of spread and a few pairs that showed indication of a rostral to caudal spread. The most that can be argued is there is an overall spread of caudal to rostral activity, not that every neuron pair shows this spread. The spread effect was also very noisy when we looked at neuron pairs saccade by saccade (Figs. 6 and 7), and this was particularly true of the peak analysis, but true of the analyses based on the whole curve of the activity (median activation and cross-correlation) as well. The issue of noise was not as serious a concern in the previous experiments (Anderson et al. 1998; Munoz and Wurtz 1995b) because the analyses were done on averaged neuronal activity. This suggests that whatever information may be conveyed by a spread of activity, it is likely to be based on changes across the population of neurons.

A third limitation is that we have provided no additional information about the direction of spread on the SC map beyond the rostral-caudal axis. Anderson et al. (1998) made the important observation that the spread of activity they observed was not limited to caudal-rostral but was directed medial- 
lateral as well. Since we hoped in this study to test whether there was a caudal to rostral shift on a saccade by saccade basis in single neuron pairs, we went to substantial effort to align our electrodes in a caudal-rostral line and did not position them to identify such lateral spread. We have no reason to doubt that such a lateral spread occurs, but if we were to study the direction of spread using simultaneous recording, the next step would seem to be alignment of multiple electrodes so as to allow comparison of caudal-rostral and medial-lateral spread. Of course all of the neurons sampled would also have to have large movement fields.

Finally, we have little evidence related to the spread of activity at the rostral pole of the SC because we studied few neurons that had open-ended movement fields in this region of the SC. When we searched for such neurons, we were struck with the paucity of neurons found in the rostral SC that had open-ended or even large movement fields. As Fig. 3 shows, we encountered no open-ended movement field neurons within the rostral $0.5 \mathrm{~mm}$ of the rostral pole of the SC. One factor may be, of course, the exclusion of neurons from our sample with tonic activity during fixation (fixation neurons) (Munoz and Wurtz 1995a); however, we had difficulty finding large movement field neurons even when we were far enough away from the rostral pole that few such fixation neurons were encountered. While various biases can certainly affect the sampling of single neurons, we devoted a substantial amount of time attempting to isolate rostral neurons with open-ended movement fields because these more rostral neurons should show the best evidence of spread. Munoz and Wurtz (1995a) alluded to this paucity but did not document it. Anderson et al. (1998) seem to have concentrated their recording largely within this region, and this may be a factor affecting the limited indication of spread that they observed.

\section{Possible functional contributions of the spread}

A number of models of saccadic control incorporate the spread of activity as the mechanism that might provide the feedback controlling when a saccade ends. These models, as they apply to the monkey, distinguish the possible roles of the burst and buildup neurons (Munoz and Wurtz 1995a). The difference in the activity between the burst neurons, which tend to have closed movement fields, and the buildup neurons, which tend to have open-ended movement fields, led to the suggestion that they play different roles in the generation of saccades (Munoz and Wurtz 1995b; Optican 1994, 1995). For example, burst neurons have been thought for some time to provide the desired change in eye position that would be executed by the impending saccade (see review by Sparks and Hartwich-Young 1989). However, a different role has been proposed for the spread of activity among the buildup neurons. In both the cat (Lefèvre and Galiana 1992; Munoz et al. 1991a) and the monkey (Munoz and Wurtz 1995b; Optican 1994), the moving hill or spread of activity, respectively, was hypothesized to convey information about the movement of the eye during its travel and termination. A related but more specific hypothesis regarding the role of this spread of activity in the monkey was advanced by Optican (1995), who suggested that it might fulfill the requirements of the re-settable integrator that is a necessary component in feedback control models of saccade generation. The support offered by the present experi- ments for a spread of activity is compatible with these models. To test these models, one must prevent or alter the spread of activity. Preventing the spread of activity should delay an integration based on the spread and cause the saccade to be too large (Optican 1995) or prevent the spread from reaching the fixation neurons at the rostral pole and stopping the current saccade (Lefèvre and Galiana 1992; Munoz and Wurtz 1995b; Munoz et al. 1991b). In either case, the saccade should continue, and the result would be a hypermetric saccade. These hypotheses have been tested by a reversible inactivation experiment (Aizawa and Wurtz 1998; Quaia et al. 1998) in which the spread of activity was disrupted by injecting muscimol into rostral locations of the superior colliculus while monkeys made saccades throughout the oculomotor range. Rather than the hypermetric saccades predicted by the hypotheses, saccades were either normometric or hypometric, although the trajectory of the saccade was altered. The lack of hypermetric saccades was taken as a clear rejection of the hypothesis. In addition, the direction of the saccade trajectories changed so that the saccade curved away from and then back toward the location of the target. This change in direction began at the start of the saccade, whereas the effect of a spread of activity should not act on the direction until late in the saccade when the spread of activity neared the rostral SC where the muscimol injection should have produced the greatest disruption. Finally, as Anderson et al. (1998) pointed out, if spread were to be essential for the control of saccadic amplitude, the spread should be most evident in the rostral SC. However, they found little indication of such a spread of activity for small saccades represented in the rostral SC, and we found few neurons that would support such a spread (those with at least large movement fields). In addition, their finding of a spread in a mediolateral direction as well as a caudal-rostral one does not support a specific functional role for the spread of activity in ending the saccade. Taken together, these observations would seem to definitively reject the hypothesis that the spread of activity controls the termination of the saccade.

We are thus in the position of providing evidence for a spread of activity for which the best formulated hypothesis for its function has at the same time been rejected. Other functions are worth considering, but since they are based on no added evidence, they are speculative.

One possibility is that the spread is in some way related to the generation of gaze shifts that involve head as well as eye movements. The observation that the spread of activity is evident with larger saccades (Munoz and Wurtz 1995b) is relevant because it is with larger gaze shifts that head movement accompanies eye movement; for gaze shifts of approximately $20^{\circ}$ or larger, eye movements are accompanied more frequently by head movements (Freedman and Sparks 1997a,b). Even when the head of the monkey is fixed, the monkey will attempt to move its head for gaze shifts larger than $20^{\circ}$ (Richmond and Wurtz 1980). We did not, however, find evidence for spread only in the caudal SC (Fig. 9). Another possibility is that the spread of activity is related to the trajectory of the saccade even though not to the termination of the saccade. In the previously mentioned reversible lesion experiments (Aizawa and Wurtz 1998; Quaia et al. 1998), a change in the curvature of the trajectory of the saccades followed muscimol injections into the SC. Since this change in direction is a shift in the relative horizontal and vertical velocities of the 
saccades, and since these injections were specifically made in such a way as to disrupt the spread of activity, the change in curvature might well result from altering the spread. Electrical stimulation of SC has also shown a relationship between the $\mathrm{SC}$ and the peak velocity of an evoked movement (Stanford et al. 1996). At this time, however, the relation of spread of activity in the monkey to either gaze or velocity is only an intriguing possibility.

Finally, it is worth noting that a spread of activity across neuronal maps is not unique to the cat and monkey SC. For example, waves of activity spreading across the retina during development have recently been documented by fluorescence imaging in ferret retina (Feller et al. 1996) as has spreading activity in the adult turtle cortex in response to visual stimulation (Prechtl et al. 1997). The role of these spatiotemporal events in either visual system development or cortical visual processing remains to be determined, as does the spread of activity in the monkey SC during individual eye movements.

We thank Dr. Christian Quaia, our colleagues in the Laboratory of Sensorimotor Research, and the two anonymous referees for comments, and T. Ruffner and N. Nichols for construction of the multielectrode microdrive. We are also grateful to H. Aizawa for early contributions in developing the multiple electrode recording techniques that we used in these experiments.

\section{REFERENCES}

AIZAWA H AND WURTZ RH. Reversible inactivation of monkey superior colliculus. I. Curvature of saccadic trajectory. J Neurophysiol 79: 20822096, 1998

Anderson RW, Keller EL, Gandhi NJ, and Das S. Two-dimensional saccade-related population activity in superior colliculus in monkey. J Neurophysiol 80: 798-817, 1998.

CRIST CF, YAmasaki DSG, Komatsu H, AND Wurtz RH. A grid system and a microsyringe for single cell recording. J Neurosci Methods 26: 117-122, 1988

Feller MB, Wellis DP, Stellwagen D, Werblin FS, and Shatz CJ. Requirement for cholinergic synaptic transmission in the propagation of spontaneous retinal waves. Science 272: 1182-1187, 1996.

FREEDMAN EG AND SPARKS DL. Activity of cells in the deeper layers of the superior colliculus of the rhesus monkey: evidence for a gaze displacement command. J Neurophysiol 78: 1669-1690, 1997a.

FREEDMAN EG AND SPARKS DL. Eye-head coordination during head-unrestrained gaze shifts in rhesus monkeys. J Neurophysiol 77: 2328-2348, 1997b.

FuCHS AF AND RoBINSON DA. A method for measuring horizontal and vertical eye movement chronically in the monkey. J Appl Physiol 21: 1068-1070, 1966.

HAYS AV, Richmond BJ, AND OPTICAN LM. A UNIX-based multiple process system for real-time data acquisition and control. WESCON Conf Proc 2: $1-10,1982$
JudGe SJ, Richmond BJ, AND ChU FC. Implantation of magnetic search coils for measurement of eye position: an improved method. Vision Res 20: $535-538,1980$

Lefèvre P AND Galiana HL. Dynamic feedback to the superior colliculus in a neural network model of the gaze control system. Neural Networks 5: 871-890, 1992

MacPherson JM AND AldRIDGe JW. A quantitative method of computer analysis of spike train data collected from behaving animals. Brain Res 175: 183-187, 1979.

MunOz DP, GuitTon D, AND PÉLISSON D. Control of orienting gaze shifts by the tectoreticulospinal system in the head-free cat. III. Spatiotemporal characteristics of phasic motor discharges. J Neurophysiol 66: 1642-1666, 1991a.

MunOz DP, PÉLisson D, AND GuitTon D. Movement of neural activity on the superior colliculus motor map during gaze shifts. Science 251: 1358-1360, $1991 b$.

MuNOZ DP AND WURTZ RH. Saccade-related activity in monkey superior colliculus. I. Characteristics of burst and buildup cells. J Neurophysiol 73: 2313-2333, 1995a.

MunOz DP AND WURTZ RH. Saccade-related activity in monkey superior colliculus. II. Spread of activity during saccades. J Neurophysiol 73: 23342348, 1995b

OptiCAN LM. Control of saccade trajectory by the superior colliculus. In: Contemporary Ocular Motor and Vestibular Research: A Tribute to David A. Robinson, edited by Fuchs AF, Brandt T, Büttner U, and Zee DS. Stuttgart: Thieme, 1994, p. 98-105.

OpTICAN LM. A field theory of saccade generation: temporal-to-spatial transform in the superior colliculus. Vision Res 35: 3313-3320, 1995.

OtTES FP, VAN Gisbergen JAM, AND EgGERMONT JJ. Visuomotor fields of the superior colliculus: a quantitative model. Vision Res 26: 857-873, 1986.

PORT NL, SOMMER MA, AND WuRTZ RH. Multi-electrode recordings in rhesus monkey superior colliculus. Soc Neurosci Abstr 24: 1498, 1998.

Prechtl JC, Cohen LB, Pesaran B, Mitra PP, and Kleinfeld D. Visual stimuli induce waves of electrical activity in turtle cortex. Proc Natl Acad Sci USA 94: 7621-7626, 1997.

Quaia C, AIZAWA H, Optican LM, AND Wurtz RH. Reversible inactivation of monkey superior colliculus. II. Maps of saccadic deficits. J Neurophysiol 79: 2097-2110, 1998.

Richmond BJ, Optican LM, Podell M, AND SPITZER H. Temporal encoding of two-dimensional patterns by single units in primate inferior temporal cortex. I. Response characteristics. J Neurophysiol 57: 132-146, 1987.

RichMOND BJ AND WURTZ RH. Vision during saccadic eye movements. II. A corollary discharge to monkey superior colliculus. J Neurophysiol 43: $1156-1167,1980$.

RoBINSON DA. Eye movements evoked by collicular stimulation in the alert monkey. Vision Res 12: 1795-1808, 1972.

SNEDECOR GW AND Cochran WG. Statistical Methods. Ames, IA: Iowa State Univ. Press, 1989.

SPARKS DL AND HARTwich-Young R. The deep layers of the superior colliculus. In: The Neurobiology of Saccadic Eye Movements, Reviews of Oculomotor Research, edited by Wurtz RH and Goldberg ME. Amsterdam: Elsevier, 1989, vol. III, p. 213-256.

Stanford TR, Freedman EG, and Sparks DL. Site and parameters of microstimulation: evidence for independent effect of the properties of saccades evoked from the primate superior colliculus. J Neurophysiol 76: 3360-3381, 1996. 